\title{
Tommaso III di Saluzzo, Le livre du Chevalier Errant (BnF ms. fr. 12599)
}

\section{Anna Maria Finoli}

\section{OpenEdition}

\section{Journals}

\section{Edizione digitale}

URL: http://journals.openedition.org/studifrancesi/6567

DOI: 10.4000/studifrancesi.6567

ISSN: 2421-5856

\section{Editore}

Rosenberg \& Sellier

\section{Edizione cartacea}

Data di pubblicazione: 1 septembre 2010

Paginazione: $344-346$

ISSN: 0039-2944

\section{Notizia bibliografica digitale}

Anna Maria Finoli, «Tommaso III di Saluzzo, Le livre du Chevalier Errant (BnF ms. fr. 12599)», Studi

Francesi [Online], 161 (LIV | II) | 2010, online dal 30 novembre 2015, consultato il 08 janvier 2021. URL: http://journals.openedition.org/studifrancesi/6567 ; DOl: https://doi.org/10.4000/studifrancesi.6567

Questo documento è stato generato automaticamente il 8 janvier 2021.

\section{(a) $\odot \Theta$}

Studi Francesi è distribuita con Licenza Creative Commons Attribuzione - Non commerciale - Non opere derivate 4.0 Internazionale. 


\title{
Tommaso III di Saluzzo, Le livre du Chevalier Errant (BnF ms. fr. 12599)
}

\author{
Anna Maria Finoli
}

\section{NOTIZIA}

TOMMASO III DI SALuzzo, Le livre du Chevalier Errant (BnF ms. fr. 12599), a cura di Marco PICCAT, Boves, Araba Fenice, 2008, 1070 pp.

1 Si tratta dell'edizione del ms. della Bibliothèque nationale de France, recentemente esposto in occasione della mostra La légende du roi Arthur (BnF, 20 ottobre 2009 - 24 gennaio 2010), preceduta da vari "interventi". Il primo, di Marco PICCAT, Tommaso III, Marchese errante: l'autobiografia cavalleresca di un Saluzzo (pp. 5-26), collocando l'opera e l'autore nella situazione del marchesato di Saluzzo alla fine del secolo xIV, con molte e pertinenti testimonianze sugli elementi biografici, sulla cultura personale di Tommaso III e quella lato sensu contemporanea del Piemonte e della Francia, costituisce una "cornice storica di riferimento" che contribuisce ad una migliore comprensione del testo. E tuttavia non si può tacere qualche dissenso. La definizione stessa di "autobiografia cavalleresca" rinvia ad una categoria di tardi romanzi in prosa definiti appunto "biografie cavalleresche", perché, diversamente dai romanzi ciclici, hanno per oggetto la storia di un solo personaggio, che dopo varie avventure raggiunge la felicità personale e uno status nella gerarchia feudale. La critica più recente, invece, ha messo in rilievo il carattere enciclopedico dell'opera, in cui si riversa la cultura dell'autore, che va dalla narrativa in versi e in prosa alla trattatistica più varia, fino alla produzione e alle tematiche del suo tempo, carattere che peraltro il Piccat riconosce nel corso dell'analisi (p. 17). Anche l'adesione incondizionata di Tommaso agli ideali cortesi è affermata in modo un po' troppo perentorio: non v'è dubbio che nella prima parte del romanzo la rappresentazione della corte d'Amore lasci trapelare la nostalgia di un mondo vagheggiato nella giovinezza, e insieme a questa irrimediabilmente perduto, ma il racconto non si esaurisce qui, il protagonista affronterà, tra l'altro, l'incontro con 
dame Fortune e le alterne, spesso tragiche vicende di molti personaggi da lei determinate, discuterà con il filosofo Raison, che argomenta con le parole stesse di Jean de Meun, non direi proprio un sostenitore della concezione cortese della vita; soprattutto concluderà il suo itinerario nella casa di Congnoissance, che ribadisce gli argomenti di Raison, gli svela il significato recondito delle sue avventure e gli inganni diabolici che si nascondono dietro le brillanti apparenze, e infine, per illustrare le virtù dei principi e dei cavalieri, espone un trattato di morale cristiana valido per tutti gli uomini.

2 Il contributo di Renato BORDONE, Une tres noble jouste (pp. 27-35), prende lo spunto dal ricordo del torneo e delle feste svoltesi a Saint-Denis in occasione dell'adoubement di Charles e Louis d'Anjou, per analizzare questo episodio storico inserito nella narrazione romanzesca e illustrarne gli aspetti araldici, ma anche per evocare momenti dei travagliati rapporti con i Savoia e segnalare gli echi della cultura francese. Il saggio, peraltro interessante e documentato, è permeato dello stesso pregiudizio che l'opera di Tommaso sia volta esclusivamente alla celebrazione della cavalleria.

3 Laura RAMELlo cura l'edizione del testo (pp. 37-43; pp. 49-553) che consiste in pratica nella trascrizione del codice parigino, BnF fr. 12599 (P), con alcuni rari emendamenti indicati nelle pagine introduttive o nell'apparato. Si tratta di un lavoro che comporta un notevole impegno, e dispiace perciò, davanti a tanta fatica, di dover esprimere delle riserve. Ora, è certamente valida la critica, anche severa, mossa dalla Ramello all'edizione di Ward (1984), il quale, pur scegliendo P come testo base, accoglie lezioni dell'altro codice che ha tràdito l'opera, il ms. L.V.6 della Biblioteca Nazionale di Torino (T), approdando così ad un testo ibrido (p. 42). Sarebbe stato comunque necessario da parte dell'editrice in primo luogo giustificare la sua scelta, necessario soprattutto seguire il suggerimento formulato già da Anna Cornagliotti («Studi Piemontesi»,VIII, 1989, pp. 3-24), e ribadito, con l'apporto di una chiara esemplificazione, da Marco Fantoni fin nel titolo di un saggio, L'importanza delle fonti in versi nella tradizione manoscritta dello Chevalier Errant di Tommaso III di Saluzzo («Medioevo Romanzo», XXIII, 1997, pp. 210-228), in altri termini, attenersi ad un criterio acquisito nella pratica ecdotica, quello dei testimoni indiretti. Non si pretende, è ovvio, che l'editore di un testo esaurisca la ricerca delle fonti prima di accingersi al suo lavoro, si può chiedere però che conosca e utilizzi almeno i risultati di altri studiosi. Un solo esempio: nel discorso di Raison citato più sopra, Amore è definito da un serie di ossimori e di antitesi, per lo più in couplets di ottonari; nel couplet "Entechiez de pardon pechiez, Pechiez de pardon entechiez" (vv. 8679-8680), il secondo verso costituisce un'inutile ripetizione; nella fonte, il Roman de la Rose, si trova la buona lezione, "De pechiez pardon entechiez", che rispetta l'antitesi. Anche in un altro caso la troppo rigida prudenza diventa veramente una manifestazione di Corruptelenkult: ai vv. 4961 e 4969 si conserva la lezione "Lamorat" in luogo di "Morholt", palese errore, testimoniato dal Gorra che in T leggeva "Morholt", senza contare che Lamorat appare ben vivo ai vv. 5251-5252, e 5313-5316. La lezione corretta è indicata solo nell'indice dei nomi e accettata nella traduzione. In entrambi i casi l'emendamento non avrebbe comportato alcun ibridismo. Qualche dubbio è suscitato dal nome della figlia di Pharamond, suicida per amore di Tristano, Gloriande, mentre è Belide nella fonte, il Roman de Tristan en prose, non "secondo la leggenda", come è detto nell'Indice dei nomi. In ordine ad un altro problema, quello della presenza di versi nella prosa, l'analisi di una bibliografia più completa avrebbe fornito alla Ramello una migliore informazione sui modi in cui 
Tommaso elabora, e talvolta ostenta, i materiali della propria cultura enciclopedica, e le avrebbe impedito di proporre emendamenti cospicui, trasformando in versi brani di prosa, sulla base di un poco filologico criterio soggettivo ("a mio avviso [...] è percettibile una musicalità tale da recuperare corrispondenze di rima, restituendo sotto forma di verso queste porzioni testuali”, p. 42). Non c'è in questa sede spazio per esaminare ad una ad una le sue proposte, di cui alcune sembrano anche probabili, ma resta il dubbio che si tratti invece di reminiscenze o allusioni ad una fonte. Rime si trovano anche in luoghi non presi in considerazione dall'editrice, ad esempio nell'episodio del gioco mondano delle demandes en amours (rr. 3190-3302), ma sono solo la spia, appunto, della fonte della casistica amorosa che è alla base del gioco, fonte costituita da raccolte di domande e risposte in versi o in prosa (edite da A. Klein nel 1911, oggetto di un'edizione critica di M. Felberg-Levitt, Montréal, 1995).

Resta da dire della traduzione di Enrica MARTINENGo (pp. 577, 581-1049). La traduttrice indica nella Nota introduttiva i criteri cui intende attenersi: "evitare il più possibile la regolarizzazione dei tempi verbali", "rispettare e conservare la varietà di livelli stilistici e lessicali [...]. In breve, non si è cercato di rendere troppo artificiosa la lettura, per mantenere la ricchezza e la precisione delle espressioni che designano oggetti, fenomeni e aspetti della vita umana" (p. 577). Non si può, naturalmente, controllare nell'intera traduzione la fedeltà ai propositi. Una rapida scorsa permette di osservare che la conservazione dei tempi verbali porta in qualche caso ad una sintassi italiana un po' claudicante, e che certe scelte, come il passaggio dal "tu" al "voi" nel primo colloquio tra il Cavaliere e Congnoissance (vv. 31-84) e altrove, o come le plaisir du doulz temps tradotto "la festa della primavera" (v. 3), appaiono incomprensibili. Qualche confronto più puntuale con il testo francese lascia ancor più perplessi proprio per quel che riguarda "la ricchezza e la precisione delle espressioni", come le pou scens [quy est en toy] che diventa "il poco senso" (v. 169) invece di "il poco senno"; baudour, "gioia, sicurezza, magnificenza", tradotto nell'autopresentazione di Cesare "trionfo" (v. 9348), termine che in bocca a lui, antico romano, assumerebbe un significato specifico; estre hostellés tradotto "essere rifocillato" (v. 288), mentre il richiamo nel testo alla stanchezza e all'ora tarda richiederebbe "essere accolto, alloggiato, ospitato", che è il primo significato di hosteller; curiosa, al limite del comico, l'interpretazione "non udire le folgori divine" (rr. 890, 1312) della locuzione ne pas oör Dieu tonnant: tale locuzione è frequentissima nella descrizione di battaglie per evocare il frastuono delle armi che impedirebbe di udire molto semplicemente un tuono (in bibliografia si cita il Dictionnaire des locutions en Moyen Français di Giuseppe Di Stefano: perché non consultarlo?).

5 Colpisce soprattutto l'inesattezza proprio nel lessico tecnico della cavalleria: adouber, variamente declinato nella cerimonia dell'adoubement (vv. 127-152), tradotto sempre con "vestire", in luogo dell'italiano "armare, creare, fare", scelta che dà poi luogo, in un'esortazione di Congnoissance, a un italiano un po' sconcertante "siete stato vestito cavaliere" (v. 160); nella stessa occasione donner la colée tradotto "colpire alla nuca" (v. 146), che evoca ben altre circostanze, mentre la colée poteva essere il colpo di spada sul collo o sulla spalla (l'italiano conosce il termine "accollata"); pallefroy non è proprio un "giovane puledro" (v. 27), bensì un cavallo robusto e tranquillo, utilizzato per viaggiare e adatto alle dame, in italiano "palafreno"; bataille può significare anche "schiera", quindi tourner la bataille arriere significherà "far arretrare la schiera" e non "la battaglia" (r. 802) come, nella riga seguente, secourir nostre bataille, "soccorrere la nostra schiera", e non genericamente "intervenire"; i belfrois non sono "palchi mobili" (v. 
5607), come gli echaffaux costruiti per assistere ai tornei, ma torri mobili assai alte per osservare dall'interno della città il campo di battaglia.

In alcuni casi, poi, manca alla traduttrice uno dei requisiti professionali fondamentali, la conoscenza dell'argomento trattato nel testo, cioè della grande narrativa romanzesca francese e dell'ideologia che la permea, ma vien da chiedersi anche quale sia il suo livello d'informazione delle strutture dell'antico e del medio francese. Ci si contenterà di citare qualche esempio. Il primo riguarda la storia di Tristan, personaggio così importante per l'Errant, e perciò tanto più grave l'incomprensione. Tommaso non è chiarissimo nel ricordare le avventure dell'eroe in Irlanda e non distingue il pericolo causato dal veleno della spada di Morholt, da cui lo salva Yseud, da quello corso quando dalla tacca nella sua spada, che corrisponde alla scheggia trovata nel corpo di Morholt, la regina, sorella di questi, riconosce in lui, rimasto anonimo fino a quel momento, l'assassino del fratello, e vorrebbe ucciderlo mentre è disarmato nel bagno: "Mais en Irlande fu il en peril voirement,/ Quant il se baingnoit, par l'envenimement/ Et Yseud la Blonde lui fu garent/ Adonc du l'osche en l'espee trouvée/ Que fist Tristan en la meslee" (vv. 4964-4968). La Martinengo non conosce l'episodio, tratto dal Roman de Tristan en prose, ignora il significato di osche e non si preoccupa di cercarlo, sorvola sulla circostanza del bagno, e interpreta: "E allora Isotta la Bionda lo curò/ Del veleno che si trovava sulla spada". Eppure nella bibliografia sono indicati alcuni autorevoli dizionari, in cui avrebbe trovato il lemma osche. Osserviamo a questo proposito che non è citato il Dictionnaire $d u$ Moyen Français (http://www.atilf.fr/dmf), certamente utile, direi indispensabile per comprendere un'opera scritta a cavallo tra il XIV e il xV secolo. Forse l'editrice stessa avrebbe dovuto accorgersi dell'incomprensibilità del testo e riflettere sulla lezione " $d u$ l'osche", clamorosa violazione delle più elementari regole morfologiche: infatti nel ms. (fol. 49va) si legge "fu l'osche", come correttamente trascrive Ward (p. 325).

7 Anche nella storia di Lancelot si devono rilevare dei vistosi fraintendimenti: nell'episodio della Douloureuse Garde che muta il nome in Joyeuse, "Pour le deduit qu'il fist leans", è inspiegabilmente tradotto "Per l'amore che vi si accese" (v. 5927), ma l'amore di Lancelot e della regina si era "acceso" da tempo, e infatti se ne parla qualche centinaio di versi prima, deduit poi non è genericamente l'amore, ma il piacere, la gioia, appunto, che nasce dalla passione appagata; in quello della Damoiselle d'Escalot (vv. 5994-6009), che ha pregato Lancelot "qu'il voulzist estre ses amis" e al suo rifiuto muore d'amore e di dolore, la Martinengo non capisce che ses amis è caso retto singolare (la declinazione a due casi non è totalmente scomparsa ai tempi di Tommaso!), crede che sia un plurale e per giustificarlo inventa un "tra" che non esiste nel testo francese, quindi traduce "di essere tra i suoi amici". La figura della Damoiselle ne esce totalmente travisata: se amis ha, come in effetti appare dal contesto, il significato di "amante", la Damoiselle che ha molti "amis" non è più il tipico personaggio, positivo, della fanciulla infelice, vittima di un unico amore impossibile, ma la dama frivola e incostante, e la sua morte apparirebbe incomprensibile.

8 I testi sono corredati da un indice tematico in italiano e da indici dei nomi propri, uno per la parte francese e uno per la traduzione, redatti con criteri diversi: ci si chiede se non si sarebbe potuto evitare il double emploi, mettendo il lettore della traduzione in grado di conoscere le notizie offerte dall'indice in francese. Infine, due pagine (pp. 1065-1066) sono occupate da un glossario italiano di cinquantasei termini, compilato in modo... "prescientifico": i lemmi sono registrati così come si trovano nel testo - 
suppongo, perché non c'è alcun riferimento numerico - senza indicazione di statuto, per cui abbiamo, tra gli altri ariana, donatista, manichei, priscillianesimo, e Pelagio, già presente nei due indici dei nomi. Era necessario, poi, spiegare a dei lettori italiani castello, contrada, drago?

9 Abbellisce il volume una serie di splendide riproduzioni delle miniature del codice: la raffinatezza della decorazione, la rappresentazione stilizzata della natura, il gioco delle forme geometriche, la vivacità e il felice accostamento dei colori negli abbigliamenti e negli oggetti compongono una rappresentazione efficace dell'aristocratica eleganza tardogotica. Avrebbero meritato qualche nota di uno specialista, e magari un indice.

10 La bellezza materiale del volume non può attenuare, comunque, il grande rammarico per l'occasione perduta, per lo sciupio di energie e di mezzi, il cui risultato è un testo non valido per l'approfondimento di un'opera così complessa e importante nella storia delle culture francese e cisalpina, e neppure corretto per l'informazione cui ha diritto anche il comune lettore. 